\title{
A MODEL FOR LIQUID FILMS IN STEAM TURBINES AND PRELIMINARY VALIDATIONS
}

\author{
Amélie Simon ${ }^{1,3}$, Meryem Marcelet ${ }^{1}$, Jean-Marc Hérard ${ }^{1,2}$, Jean-Marc Dorey ${ }^{1}$, Michel Lance ${ }^{3}$ \\ ${ }^{1}$ EDF R\&D - MFEE - 6 quai watier, 78400, Chatou, France \\ 2 I2M - UMR CNRS 7373 - F13453 Marseille, France \\ ${ }^{3}$ LMFA - UMR CNRS 5509 - Ecole Centrale de Lyon, 69134, Ecully, France
}

\begin{abstract}
Liquid films in steam turbines, present in usual operating conditions, play a large but poorly understood part in the wetness-born troubles (power losses and erosion). More knowledge is needed to estimate their impacts and lessen their effects. The aim of this paper is to propose and verify a model to predict these liquid films. This model is based on modified ShallowWater equations (integral formulation). It takes into account inertia, mass transfer, gravity, gas and wall frictions, pressure, surface tension, droplet impacts, rotational effects and is unsteady. A $2 D$ code has been developed to implement this model. A part of the model has been verified with analytical solutions (Riemann problems and inclined lake at rest), has been confronted with the linear stability of falling liquid film and has been validated with the experiment of Hammitt et al. [1] which involves a sheared film under low-pressure steam turbine conditions.
\end{abstract}

\section{INTRODUCTION}

It is well known that wetness in steam turbines involves a cascade of phenomena: during the rapid expansion of steam in turbines, the saturation line is crossed and droplets are generated either by heterogeneous condensation (condensation on pre-existing nuclei in the steam) or homogeneous nucleation (pure water new nuclei generated in the absence of pre-existing nuclei). These droplets are carried downstream by steam flow, grow and a part of them deposit on blades, thus creating liquid films. These liquid films induce additional friction losses. Furthermore, these films atomize at free surfaces and at geometric edges generating bigger drops than nucleation droplets. These large drops, striking the blades located downstream, cause erosion and braking losses. These phenomena driving liquid films on steam turbine blades are summed up on figure 1. Concerning the mass balance, these films are created by the deposition of nucleation droplets, atomized drops and condensation, and are reduced by the film atomization, and evaporation. Regarding force balance, these films undergo gravity, free surface friction, wall friction, steam pressure, surface tension, droplet impact and rotation effects for the rotor blade.

Coping with liquid films is of central concern to reduce the damaging consequences of wetness in steam turbines. Nowadays no well fitted model exists to describe these liquid films. Building a more comprehensive model is necessary, first, to understand how these films are generated and behave (streamlines, thickness and velocity). Second, to estimate losses: these liquid films are at the origin of an increase of friction, of the load increase on moving blades, of generating coarse drops, of possible changes in lift blades, etc. This more comprehensive model could eventually be used by steam turbine designers to remove or reduce these liquid films by positioning suction slots on the fixed blades, special trailing edge patterns, tailor-made bleedings or heating process. It could also be used by steam turbine operators to control liquid films malfunctioning, such as vibration crisis due to film cooling on a part of the shaft [2] or stress corrosion cracking due to liquid films on the hub. Although this paper focuses on liquid films on steam turbine blades, the proposed model is more general and could be used on casing or on diffuser surfaces. 


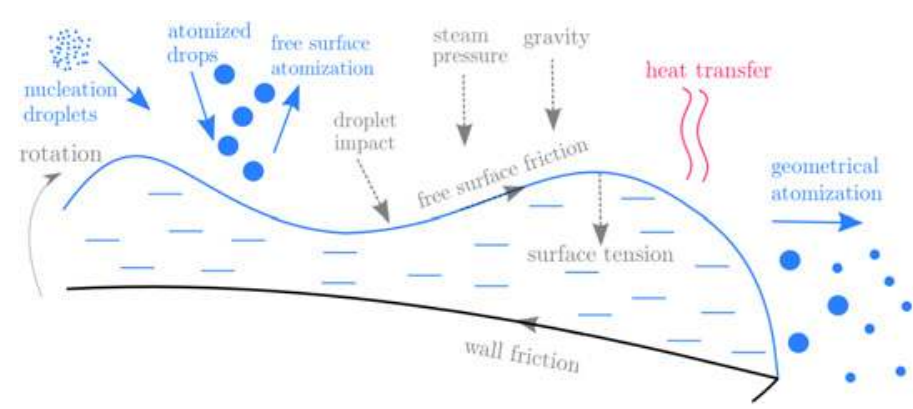

FIGURE 1. LIQUID FILM ON STEAM TURBINE BLADES

To represent this liquid film on steam turbine blades, a few approaches have been developed in the past. Concerning stator's model (with no rotation), Hammitt et al. [3] assume that the interfacial shear is predominant, and, by means of experimental considerations for the friction at the interface, they build a stationary Couette model. Gyarmathy [4] uses the same theoretical approach but includes the droplet impact. Kirillov and Yablonik [5] do not consider the droplet impact but add the pressure of the gas. Malamatenios et al. [6] significantly improve these latter models by considering inertia, wall shear and gravity with an integral formulation. Concerning rotor's model (with rotation), a stationary approach is proposed by Gyarmathy [4] taking only the centrifugal forces into account. Then, an unsteady model is presented by Kirillov and Yablonik [5] with inertia, wall shear, centrifugal and Coriolis forces. Williams and Young [7] improve it by adding the shear stress at the free surface and the pressure gradient, and by specifying the friction coefficient at the wall. Using an integral formulation, Schuster et al. [8] add at the latter model mass transfer and droplet impact.

However due to the simplifying assumptions adopted, all these models are unable to reproduce correctly the generation of waves in the liquid film and hence to describe accurately either the friction or the early stages before atomization. The goal of this paper is to present an instantaneous and more comprehensive model for liquid films on both stator and rotor blades in steam turbines. This one takes into account more physical phenomena than former ones, for instance, the surface tension and gravity compared to Schuster et al.'s model. The surface tension could play an important role in the dynamics of films because of their thinness, the gravity as well for configuration where films are subjected to inverted gravity. Particular attention is paid to justify the assumptions made to obtain this model, including the development and the closure laws. Compared to former models, verifications and validations for a part of the model are presented. Although more studies are needed to conclude, the model and the code show encouraging results.

\section{LIQUID FILM MODEL}

In the light of past studies, a good compromise to obtain the instantaneous height and velocity of thin, laminar and continuous liquid films, which is assumed for steam turbines, is an integral formulation of simplified Navier-Stokes equations. The most famous one is the Shallow-Water (or Saint-Venant) equations which are widely applied for natural flows. The authors propose to modify the Shallow-Water equations to represent steam turbine liquid films. These modifications consist in taking specific physical phenomena into account compared to the classical Shallow-Water model, such as mass transfer, gas pressure and friction, surface tension and rotation. In a first attempt, this model does not take into account the atomization of the liquid film and the blade is represented as a flat plate aligned with the turbine shaft. To construct this model additional terms linked to our particular physics are first added to the full incompressible Navier-Stokes equations. These costly equations are simplified by neglecting the terms with a lower order of magnitude. To estimate the global liquid film behaviour, these equations are then integrated through the thickness of the liquid film. This inherently leads to a loss of information inside the film. Indeed, each section of the liquid film is described by its mean height and mean velocity. During this process, some terms cannot be calculated exactly, so that the final step consists in determining closure laws.

\section{Frames of reference}

The incompressible Navier-Stokes equations are written in the local frame relative to the blade $(\vec{x}, \vec{y}, \vec{z})$ which is sketched as a flat plate as illustrated on figure 2 . The frame $\left(\overrightarrow{x_{0}}, \overrightarrow{y_{0}}, \overrightarrow{z_{0}}\right)$ is associated with the turbine shaft. The rotational vector $\vec{\Omega}$ is collinear to the axis $\overrightarrow{x_{0}}$ and its value $\omega_{0}$, is constant. In this paper we consider the case where $\overrightarrow{x_{0}}$ depends on $\vec{x}$ only. In other words, this study is restricted to flat plates in the axis of the turbine shaft. The radius of the turbine shaft is $r$. Another local frame is introduced to describe the free surface of the liquid film, $\left(\overrightarrow{t_{x}}, \overrightarrow{t_{y}}, \vec{n}\right)$ and is drawn on figure 2. The height of the film is $h$ and the position of the free surface is $\eta$. One should pay attention to the projection of the gravity on the local frame $(\vec{x}, \vec{y}, \vec{z})$.

$$
g_{x}=\vec{g} \cdot \vec{x}=0 ; g_{y}=\vec{g} \cdot \vec{y}=g \sin \theta ; g_{z}=\vec{g} \cdot \vec{z}=-g \cos \theta
$$

\section{Boundary conditions}

Let us suppose that liquid films stick to the wall, in other words that no-slip boundary condition is applied:

$$
u_{\left.\right|_{z=0}}=0 ; v_{\left.\right|_{z=0}}=0 ; w_{\left.\right|_{z=0}}=0
$$

with $\mathrm{u}, \mathrm{v}$ and $\mathrm{w}$ the velocities relative to the blade respectively in the $\vec{x}, \vec{y}$ and $\vec{z}$ directions.

We also need a way to take mass transfer (deposition, atomiza- 

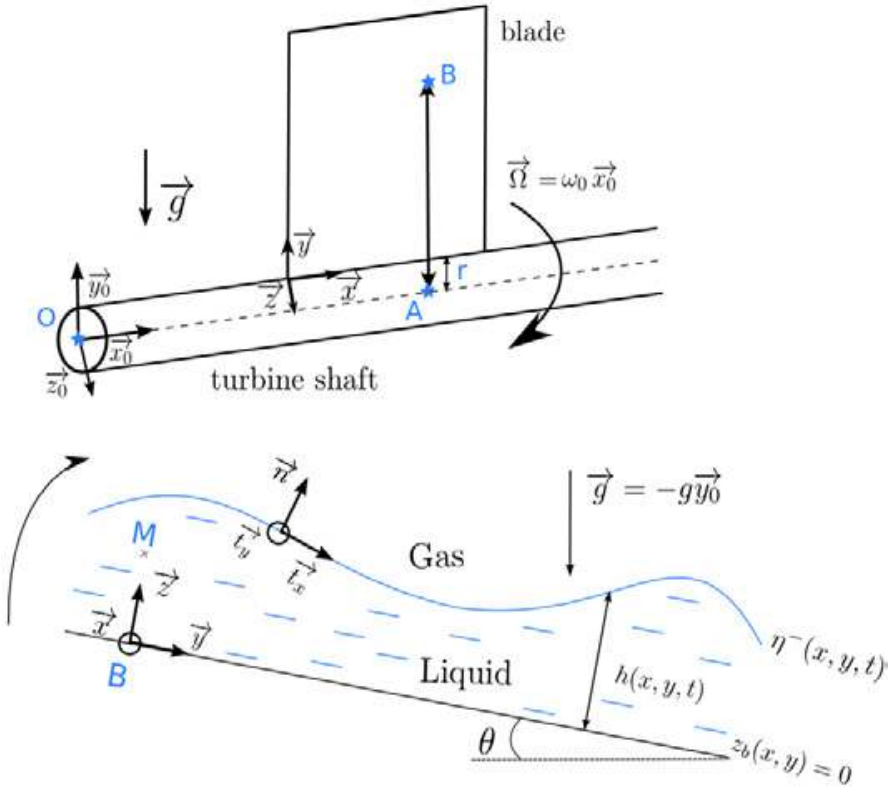

FIGURE 2. ABSOLUTE AND LOCAL FRAMES

tion, condensation and evaporation) into account. A mass balance on an infinitesimal volume at the free surface gives:

$$
\frac{d\left(\eta^{-}-z\right)}{d t}=S_{m}
$$

with $S_{m}$ the mass transfer. The material derivative of $\left(\eta^{-}-z\right)$ represents the motion of a particle following the free surface. Expression (3) translates the fact that for incompressible flows, the position of the free surface depends on the mass transfer. The Leibniz equation for moving integral borders is used to split this derivation and obtain the free surface boundary condition with mass transfer.

$$
\left.w\right|_{z=\eta^{-}}=\partial_{t} \eta^{-}+u_{\left.\right|_{z=\eta^{-}}} \partial_{x} \eta^{-}+v_{\left.\right|_{z=\eta^{-}}} \partial_{y} \eta^{-}-S_{m}
$$

\section{Simplified Navier-Stokes equations}

We consider the unsteady Navier-Stokes equations for incompressible flows in cartesian coordinates. The model is written in the local frame related to the flat plate, thus the velocity is relative to the wall. The gravity terms are displayed in equation (1). In the relative frame detailed on figure 2 , the rotational effect induces centrifugal and Coriolis forces in the $\vec{y}$ and $\vec{z}$ directions. For a thin film, the ratio of height to length, called $\varepsilon$, is small. By doing an asymptotic development with respect to $\varepsilon$, one can compare terms between them [9]. Since only mean values are accounted for, we choose to keep the horizontal convection of the film and neglect the vertical convection throughout the liquid film. This choice brings us to neglect terms above order $\mathscr{O}\left(\varepsilon^{2}\right)$, except for the rotational terms. It is of major importance to keep all of them to establish an entropy equation for this model [9]. One should not be surprised not to see a mass term in the continuity equation (or a droplet impact effect in the momentum equations) since within the liquid film volume there is no mass transfer. The simplified Navier-Stokes equations at order $\mathscr{O}(\varepsilon)$ finally read:

$$
\begin{aligned}
& \frac{\partial u}{\partial x}+\frac{\partial v}{\partial y}+\frac{\partial w}{\partial z}=0 \\
& \frac{\partial u}{\partial t}+\frac{\partial u^{2}}{\partial x}+\frac{\partial u v}{\partial y}+\frac{\partial u w}{\partial z}=-\frac{1}{\rho} \frac{\partial p}{\partial x}+\frac{1}{\rho} \frac{\partial \tau_{x z-r}}{\partial z} \\
& \frac{\partial v}{\partial t}+\frac{\partial u v}{\partial x}+\frac{\partial v^{2}}{\partial y}+\frac{\partial v w}{\partial z}=g_{y}-\frac{1}{\rho} \frac{\partial p}{\partial y}+\frac{1}{\rho} \frac{\partial \tau_{y z-r}}{\partial z}+\omega_{0}^{2}(r+y)+2 \omega_{0} w \\
& \frac{\partial p}{\partial z}=\rho g_{z}-2 \rho \omega_{0} v+\rho \omega_{0}^{2} z
\end{aligned}
$$

with $p$, the pressure, $\rho$ the density, $\tau_{x z-r}=\mu \partial u / \partial z$ and $\tau_{y z-r}=$ $\mu \partial v / \partial z$, the reduced viscous shear stresses at order $\mathscr{O}(\varepsilon)$.

\section{Integral formulation}

To compute the average height and velocity at any position of the liquid film, we integrate the simplified Navier-Stokes equations (5) over the film thickness. Let us define the mean value of $x$, where $x$, stands for $u, v, w$ or $h$ :

$$
\bar{x}=\frac{1}{h} \int_{0}^{\eta^{-}} x d z ; h=\int_{0}^{\eta^{-}} d z
$$

By applying the Leibniz rule and using the boundary condition defined in equation (2), the continuity equation becomes:

$$
\frac{\partial}{\partial x} \int_{0}^{\eta^{-}} u d z-u_{\left.\right|_{\eta^{-}}} \frac{\partial \eta^{-}}{\partial x}+\frac{\partial}{\partial y} \int_{0}^{\eta^{-}} v d z-v_{\left.\right|_{\eta^{-}}} \frac{\partial \eta^{-}}{\partial y}+w_{\left.\right|_{\eta^{-}}}=0
$$

The kinematic boundary condition (equation (4)) and the definition of the average variable (equation (6)) result in:

$$
\frac{\partial h}{\partial t}+\frac{\partial h \bar{u}}{\partial x}+\frac{\partial h \bar{v}}{\partial y}=S_{m}
$$

By integrating the $z$-momentum equation, we obtain the pressure field within the film. The Coriolis related term cannot be determined exactly as the integration does not cover the entire thickness of the film. We note $p\left(x, y, \eta^{-}, t\right)=\mathscr{P}_{\left.\right|_{z=\eta^{-}} ^{\text {liquid }}}(x, y, t)$.

$$
\begin{aligned}
p(x, y, z, t) & =\mathscr{P}_{\left.\right|_{z=\eta^{-}} ^{\text {liquid }}}(x, y, t)+\rho g_{z}(z-h(x, y, t)) \\
& +2 \rho \omega_{0} \int_{z}^{\eta^{-}} v d z+\frac{\rho \omega_{0}^{2}}{2}\left(z^{2}-h(x, y, t)^{2}\right)
\end{aligned}
$$

By comparing with the Shallow-Water equations, one can remark that the pressure is not only hydrostatic but also depends on rotational effects. Proceeding in the same way as for the continuity equation for the left hand side term and replacing the pressure 
with the integrated $z$-momentum equation (9), we express the $x$ momentum equation:

$$
\begin{aligned}
& \frac{\partial h \bar{u}}{\partial t}+\frac{\partial}{\partial x} \int_{0}^{\eta^{-}} u^{2} d z+\frac{\partial}{\partial y} \int_{0}^{\eta^{-}} u v d z=-\frac{h}{\rho} \frac{\partial \mathscr{P}_{\left.\right|_{z=\eta^{-}}}}{\partial x}+h g_{z} \frac{\partial \eta^{-}}{\partial x}+\frac{\omega_{0}^{2}}{2} h \frac{\partial \eta^{-2}}{\partial x} \\
& -2 \omega_{0} \int_{0}^{\eta^{-}} \frac{\partial \int_{z}^{\eta^{-}} v d z}{\partial x} d z+\frac{1}{\rho}\left(\tau_{x z-\left.r\right|_{\eta^{-}}}-\tau_{x z-\left.r\right|_{0}}\right)+u_{\left.\right|_{\eta^{-}}} S_{m}
\end{aligned}
$$

A cautionary remark is to be made concerning the droplet impact represented by the term $u_{\left.\right|_{\eta^{-}}} S_{m}$. This one does not need to be added to the equation (5) since it appears naturally during the integration step as long as there is a mass transfer in the kinematic boundary condition at the free surface (4).

\section{Exact expression}

There is no other difficulty to express the $y$-momentum equation. Eventually, the exact expression for the integrated model is:

$$
\frac{\partial h}{\partial t}+\frac{\partial h \bar{u}}{\partial x}+\frac{\partial h \bar{v}}{\partial y}=S_{m}
$$

$$
\begin{aligned}
& \frac{\partial h \bar{u}}{\partial t}+\frac{\partial}{\partial x}\left(\int_{0}^{\eta^{-}} u^{2} d z+\frac{g \cos \theta h^{2}}{2}\right)+\frac{\partial}{\partial y} \int_{0}^{\eta^{-}} u v d z+2 \omega_{0} \int_{0}^{\eta^{-}} \frac{\partial \int_{z}^{\eta^{-}} v d z}{\partial x} d z \\
& -\frac{\omega_{0}^{2}}{2} h \frac{\partial \eta^{2}}{\partial x}=-\frac{h}{\rho} \frac{\partial \mathscr{P}_{\left.\right|_{z=\eta^{-}}}^{\text {liquid }}}{\partial x}+\frac{1}{\rho}\left(\tau_{x z-\left.r\right|_{\eta^{-}}}-\tau_{x z-\left.r\right|_{0}}\right)+u_{\left.\right|_{\eta^{-}}} S_{m}
\end{aligned}
$$

$$
\begin{aligned}
& \frac{\partial h \bar{v}}{\partial t}+\frac{\partial}{\partial x} \int_{0}^{\eta^{-}} u v d z+\frac{\partial}{\partial y}\left(i n t_{0}^{\eta^{-}} v^{2} d z+\frac{g \cos \theta h^{2}}{2}\right)+2 \omega_{0} \int_{0}^{\eta^{-}} \frac{\partial \int_{z}^{\eta^{-}} v d z}{\partial y} d z \\
& -\frac{\omega_{0}^{2}}{2} h \frac{\partial \eta^{2}}{\partial y}-2 \omega_{0} h \bar{w}=g \sin \theta h-\frac{h}{\rho} \frac{\partial \mathscr{P}_{\left.\right|_{z=\eta^{-}}}}{\partial y}+\frac{1}{\rho}\left(\left.\tau_{y z-r}\right|_{\eta^{-}}-\left.\tau_{y z-r}\right|_{0}\right) \\
& +h \omega_{0}^{2}(r+y)+v_{\left.\right|_{\eta^{-}}} S_{m}
\end{aligned}
$$

\section{CLOSURE LAWS}

To complete the system of equations (11) to (13), eight terms need closure laws.

\section{Pressure at the free surface}

Let's begin with the pressure of the film at the free surface $\mathscr{P}_{\left.\right|_{z=\eta^{-}} \text {liquid }}$ At the free surface, a density discontinuity (liquid-gas for example) leads to a depopulation of liquid molecules. This induces unbalance between attraction and repulsion forces. The part of the attraction forces which is not offset by the repulsion forces is tangential to the free surface and is called the surface tension. For a thin film $(10 \mu \mathrm{m}$ to $100 \mu \mathrm{m}$ [3], [10]), the surface tension could play an important role in its dynamics. By adding and subtracting the gas pressure at the free surface, we choose to express the surface tension by means of Laplace law. This assumption neglects the viscous effects in the normal direction of the free surface:

$$
\begin{aligned}
& \mathscr{P}_{\left.\right|_{z=\eta^{-}} ^{\text {liquid }}}=\mathscr{P}_{\left.\right|_{z=\eta^{+}} ^{\text {gas }}}+\mathscr{P}_{\left.\right|_{z=\eta^{-}} ^{\text {liquid }}}-\mathscr{P}_{\left.\right|_{z=\eta^{+}} ^{\text {gas }}} \\
& \mathscr{P}_{\left.\right|_{z=\eta^{-}} \text {liquid }}=\mathscr{P}_{\left.\right|_{z=\eta^{+}} ^{\text {gas }}}+\frac{\sigma}{\mathscr{R}}
\end{aligned}
$$

with $1 / \mathscr{R}$, the curvature at the free surface and $\sigma$, the surface tension coefficient. For surfaces described by $z=\eta(x, y)$ in the frame related to the blade, we have [11], [12]:

$$
\frac{1}{\mathscr{R}}=-\frac{\partial_{x x} \eta\left(1+\left(\partial_{y} \eta\right)^{2}\right)+\partial_{y y} \eta\left(1+\left(\partial_{x} \eta\right)^{2}\right)-2 \partial_{x x} \eta \partial_{x} \eta \partial_{y} \eta}{\left(1+\left(\partial_{x} \eta\right)^{2}+\left(\partial_{y} \eta\right)^{2}\right)^{3 / 2}}
$$

With the expression of the curvature (15), the higher order term of the surface tension appears at order $\mathscr{O}\left(\varepsilon^{2}\right)$ in the global model. Thus, the surface tension should be neglected as the simplified model (5) is built at order $\mathscr{O}(\varepsilon)$. However, Ruyer-Quil and Manneville [13] did a meaningful study on the effect of the surface tension for falling films. They conclude that surface tension contributes to the evolution of the pressure at the free surface at order $\mathscr{O}(0)$ and thus we have to take the term involving the surface tension into account at an earlier expansion stage. Therefore, the expression of the curvature (15) involving the surface tension at order $\mathscr{O}(\varepsilon)$ in the global model is kept:

$$
\frac{1}{\mathscr{R}}=-\left(\partial_{x x} \eta^{-}+\partial_{y y} \eta^{-}\right)
$$

The value $\mathscr{P}_{\mid z=\eta^{+}}$e.g. the pressure of the gas in the turbine, is prescribed in a chain manner, using external aerothermodynamic calculations of the expansion of the steam [10], [14].

\section{Shear stress}

Many investigations have been done to establish the expressions of shear stress at the wall and at the free surface and some relevant ones were retained for our study.

At the wall Classical dimensional analysis shows that the wall shear stress $\overrightarrow{\tau_{w}}=\left(\tau_{x z-\left.r\right|_{0}},\left.\tau_{y z-r}\right|_{0}\right)^{T}$ reads:

$$
\overrightarrow{\tau_{w}}=\frac{c_{f} \rho|\overrightarrow{\vec{u}}| \overrightarrow{\vec{u}}}{2}
$$

with $c_{f}$ the friction coefficient and $\overrightarrow{\vec{u}}=(\bar{u}, \bar{v})^{T}$ the mean velocity relative to the blade. For a Poiseuille flow, it is common to take

$$
c_{f}=\frac{16}{R e}
$$

Spedding and Hand [15] propose to use the following friction coefficient which better fits their experiments for smooth and wavy interfaces:

$$
c_{f}=\frac{24}{R e}
$$


Another method to express the wall shear is to set a parabolic velocity profile [16], [17], [10]. Legitimacy considerations for this closure are presented in paragraph Non-linear terms. By doing an asymptotic development with respect to the velocity, the wall shear, with a free surface shear as boundary condition, writes:

$$
\vec{\tau}_{w}=\frac{3 \mu}{h} \overrightarrow{\vec{u}}-\frac{\vec{\tau}_{\text {interface }}}{2}
$$

with

$$
\overrightarrow{\vec{u}}=\frac{1}{h} \int_{0}^{h} \vec{u}(z) d z ; \overrightarrow{\tau_{w}}=\left.\frac{\partial \vec{u}}{\partial z}\right|_{z=0} ; \vec{\tau}_{\text {interface }}=\left.\frac{\partial \vec{u}}{\partial z}\right|_{z=h}
$$

At the free surface In order to obtain the shear stress at the free surface $\vec{\tau}_{\text {interface }}=\left(\tau_{x z-\left.r\right|_{\eta^{-}}},\left.\tau_{y z-r}\right|_{\eta^{-}}\right)^{T}$, we use the continuity of forces at interface:

$$
\vec{\Sigma} \cdot \vec{n}_{\left.\right|_{z=\eta^{-}} ^{f i l m}}=\vec{\Sigma} \cdot \vec{n}_{\left.\right|_{z=\eta^{+}} ^{\text {gas }}}
$$

with $\vec{n}=\left(-\partial_{x} \eta,-\partial_{y} \eta, 1\right)^{T} / \sqrt{1+|\nabla \eta|^{2}}$ and $|\nabla \eta|^{2}=\left(\partial_{x} \eta\right)^{2}+$ $\left(\partial_{y} \eta\right)^{2}$ After projecting the equation (22) on the tangential free surface frame $\left(\overrightarrow{t_{x}}, \overrightarrow{t_{y}}\right)$, we compare the order of magnitude of the non-dimensional terms and neglect terms of order $\mathscr{O}\left(\varepsilon^{2}\right)$. We obtain:

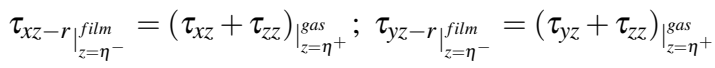

The right hand side expressions of the equations (23) require closure laws. Most of the studies assimilates the free surface with waves as a fully rough solid wall. By utilizing the same form as the equation (17):

$$
\vec{\tau}_{\text {interface }}=\frac{c_{f} \rho_{g}\left|\overrightarrow{u_{g}}-\overrightarrow{\vec{u}}\right|\left(\overrightarrow{u_{g}}-\overrightarrow{\vec{u}}\right)}{2}
$$

with $g$ the subscript for gas. The difficulty lies in the determination of $c_{f}$. Several authors notice that waves at the free surface increase significantly friction [18], [19], [20], [3]. For classical Shallow-Water equations for natural flows, the wind force induces friction whose $c_{f}$ is prescribed as follows [21]:

$$
\begin{array}{ll}
\text { for } \vec{u}_{\text {wind }}<5 \mathrm{~m} / \mathrm{s} & c_{f}=1.13 \times 10^{-3} \\
\text { for } \vec{u}_{\text {wind }}>20 \mathrm{~m} / \mathrm{s} & c_{f}=5.03 \times 10^{-3}
\end{array}
$$

Cohen and Hanratty [22] observe for two-dimensional small amplitude waves that:

$$
c_{f}=0.0142
$$

Miya et al. [12] correlate the friction coefficient for a free surface with roll-waves assuming that $R e<1000$ :

$$
c_{f}=0.008+2 \times 10^{-5} \operatorname{Re}
$$

- Crane, 1973 Young,1988

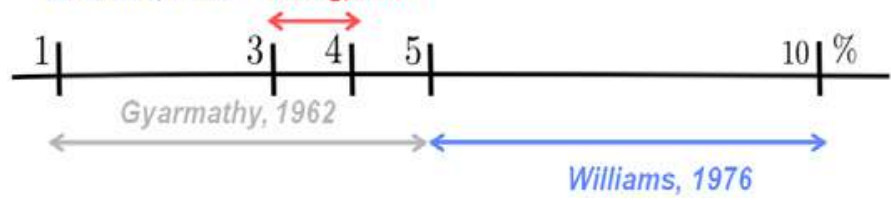

FIGURE 3. MASS PERCENTAGE DEPOSITION OF NUCLEATION DROPLETS IN LP STEAM TURBINES [25], [26], [4], [27]

with $R e$, the Reynolds number of the liquid phase. Ihnatowicz et al. [23] carry out experimental and theoretical studies to express $c_{f}$ for liquid films in steam turbines [20], [10]:

$$
c_{f}=\left(0.0007+0.0625 R e_{g}^{-0.32}\right)(1+0.025 R e)
$$

with $R e_{g}$, the Reynolds number of the gas phase.

\section{Velocity at the free surface}

Special attention was paid to determine the velocity $u_{\left.\right|_{\eta^{-}}}$and $v_{\left.\right|_{\eta^{-}}}$coming with the mass transfer. In order to better understand these terms, the authors construct the energy equation (or mathematical entropy) of a global system composed with droplets and gas using a statistic two-fluid model. Stating that the entropy of the global system increases with time and also that the mass transfer between the two phases is only due to thermodynamic phenomena, it follows that the only entropy-consistent velocities are:

$$
u_{\left.\right|_{\eta^{-}}}=\frac{u_{\text {liquid }}+u_{\text {gas }}}{2} ; v_{\left.\right|_{\eta^{-}}}=\frac{v_{\text {liquid }}+v_{\text {gas }}}{2}
$$

We eventually determine our velocity closures by assuming that $u_{\text {liquid }}=\bar{u}$ and $v_{\text {liquid }}=\bar{v}$. To ensure that this model matches expected physical behaviour, we may consider that the gas and the liquid flow are at the same speed. The only way for the free surface to depend on both phases and to have the same velocity as both phases, is by assuming this velocity to be equal to the average gas-liquid velocity.

\section{Mass term}

In a first attempt to close the term $S_{m}$, we assume that the liquid film is only fed by nucleation droplets and once these droplets deposit, they fully adhere to the blades [24], [16], [8]. To determine $S_{m}$, the droplet field and the deposition rate are needed. The first approach uses relevant literature results gathered in Fendler's thesis [10]. In all the studies shown in figure 3, the deposition appears to represent $1 \%$ to $10 \%$ of the total liquid mass in the steam. By setting a classical steam turbine wetness (up to $15 \%$ ) and steam flow rate, a value of the mass term can be estimated. Another more precise approach consists in extracting the local droplet number and radius from the steam field resolved 


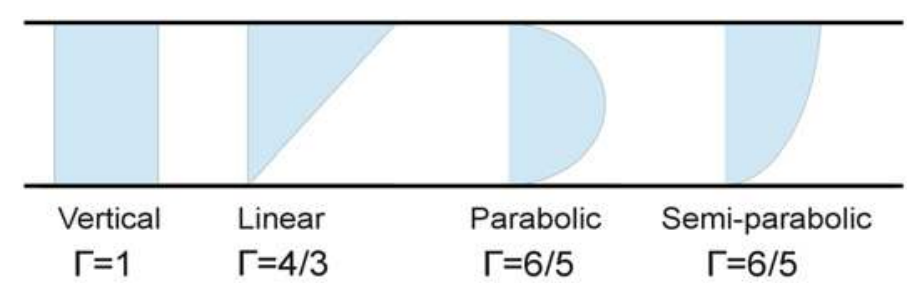

FIGURE 4. VELOCITY PROFILE WITH PROFILE FACTOR

for a wet steam turbine [10], [14], and to translate these data into a deposition rate using Zaichik's correlation [28].

\section{Non-linear terms}

Another exact way to write the non-linear terms $\int_{0}^{\eta^{-}} u^{2} d z, \int_{0}^{\eta^{-}} u v d z$ and $\int_{0}^{\eta^{-}} v^{2} d z$, is:

$$
\begin{aligned}
& \int_{0}^{\eta^{-}} u^{2} d z=h \bar{u}^{2}+\int_{0}^{\eta^{-}} u^{\prime 2} d z ; \int_{0}^{\eta^{-}} v^{2} d z=h \bar{v}^{2}+\int_{0}^{\eta^{-}} v^{\prime 2} d z \\
& \int_{0}^{\eta^{-}} u v d z=h \bar{u} \bar{v}+\int_{0}^{\eta^{-}} u^{\prime} v^{\prime} d z
\end{aligned}
$$

An interesting similarity can be made with turbulence. Statistically averaging or integrating the Navier-Stokes equations makes fluctuation terms appear. In the first case, they represent turbulence and in the second case, they represent dispersion. The latter is convection due to the local velocity inhomogeneity.

The simplest closure for the non-linear term is to assume that the integrated fluctuations, or deviations from the mean, are equal to zero. They vanish exactly either by setting a vertical velocity profile or by minimizing the energy system, as proposed by Godlewski and Sainte-Marie [29]. Besides, for a theoretical stationary viscous sheared flow under pressure gradient (the Couette and Poiseuille flow), the profile is parabolic. Moreover, the experiments of Bertschy [30], Alekseenko [31] and Adomeit [32] of falling liquid films reveal an instantaneous parabolic profile. Under these considerations, another possible closure is to set a parabolic velocity profile. These two closures are summed up in the following equation (31) by introducing the constant $\Gamma$ called the profile factor: $\Gamma=1$ for vertical profile and $\Gamma=6 / 5$ for parabolic profile (no matter the slope). Figure 4 shows different velocity profiles and their corresponding profile factors.

$$
\int_{0}^{\eta^{-}} u^{2} d z=\Gamma h \bar{u}^{2} ; \int_{0}^{\eta^{-}} u v d z=\Gamma h \bar{u} \bar{v} ; \int_{0}^{\eta^{-}} v^{2} d z=\Gamma h \bar{v}^{2}
$$

\section{Coriolis effect}

Up to this point, we have not talked about rotational forces. The Coriolis effect introduces an integration over a partial thickness, $\int_{z}^{\eta^{-}} v d z$ which cannot be solved exactly. For more clarity, we split the term as follows:

$$
\int_{z}^{\eta^{-}} v d z=\bar{v}\left(\eta^{-}-z\right)+\int_{z}^{\eta^{-}} v^{\prime} d z
$$

Like Dellar et al. [33] in their asymptotic development for a thin film under rotation, we neglect $\int_{z}^{\eta^{-}} v^{\prime} d z$. The Coriolis effect gives birth to another undetermined expression. As we no longer take into account the $z$-momentum equation, we need a formula for $\bar{w}$. A possibility is to link the velocity components using the continuity equation, boundary conditions and the Leibniz equation [29]. Once again, we neglect the integration of the deviations from the mean over a partial thickness which leads to:

$$
\bar{w}=-\frac{h}{2}\left(\frac{\partial \bar{u}}{\partial x}+\frac{\partial \bar{v}}{\partial y}\right)
$$

Nonetheless, a cautionary remark has to be done concerning the consistency between the closure of the Coriolis and the nonlinear terms. If there is a Coriolis effect, the integrated deviation from the mean for the non-linear terms must also be zero. In practice, for the rotor model, $\Gamma=1$ and for the stator model $\Gamma=1$ or $6 / 5$.

\section{General liquid film model}

Finally, the model for stator $\left(\omega_{0}=0\right)$ and rotor proposed in this paper is the system (34) to (36).

$$
\begin{aligned}
& \frac{\partial h}{\partial t}+\frac{\partial h \bar{u}}{\partial x}+\frac{\partial h \bar{v}}{\partial y}=S_{m} \\
& \frac{\partial h \bar{u}}{\partial t}+\frac{\partial}{\partial x}\left(\Gamma h \bar{u}^{2}+\frac{g \cos \theta h^{2}}{2}\right)+\frac{\partial \Gamma h \bar{u} \bar{v}}{\partial y}+2 \omega_{0}\left(\bar{v} h \frac{\partial h}{\partial x}+\frac{h^{2}}{2} \frac{\partial \bar{v}}{\partial x}\right)-\frac{\omega_{0}^{2}}{2} h \frac{\partial h^{2}}{\partial x} \\
& =-\frac{h}{\rho} \frac{\partial \mathscr{P}_{z=h^{\text {gas }}}}{\partial x}-\frac{\sigma h}{\rho} \frac{\partial \mathscr{R}^{-1}}{\partial x}+\frac{1}{\rho}\left(\tau_{x z-\left.r\right|_{\eta}}-\tau_{x z-\left.r\right|_{0}}\right)+\left(\frac{\bar{u}+u_{g a s}}{2}\right) S_{m} \\
& \frac{\partial h \bar{v}}{\partial t}+\frac{\partial \Gamma h \bar{u} \bar{v}}{\partial x}+\frac{\partial}{\partial y}\left(\Gamma h \bar{v}^{2}+\frac{g \cos \theta h^{2}}{2}\right)+2 \omega_{0}\left(\bar{v} h \frac{\partial h}{\partial y}+\frac{h^{2}}{2} \frac{\partial \bar{v}}{\partial y}\right)-\frac{\omega_{0}^{2}}{2} h \frac{\partial h^{2}}{\partial y} \\
& +2 \omega_{0} \frac{h^{2}}{2}\left(\frac{\partial \bar{u}}{\partial x}+\frac{\partial \bar{v}}{\partial y}\right)=g \sin \theta h-\frac{h}{\rho} \frac{\partial \mathscr{P}_{\left.\right|_{z=h^{+}}}}{\partial y}-\frac{\sigma h}{\rho} \frac{\partial \mathscr{R}{ }^{-1}}{\partial y}+h \omega_{0}^{2}(r+y) \\
& +\frac{1}{\rho}\left(\tau_{y z-r}-\tau_{y z-r}\right)+\left(\frac{\bar{v}+v_{g a s}}{2}\right) S_{m}
\end{aligned}
$$

The boundary conditions are the no-slip boundary at the wall (equation (2)), the kinematic boundary at the free surface (equation (4)) and the continuity of forces at the interface (equation (22)).

For this model, hyperbolicity and mathematical entropy conditions have been established as well as Galilean and rotational invariance conditions [9]. 


\section{MODEL VERIFICATIONS AND VALIDATIONS}

An independent 2D code for unstructured meshes has been developed using finite volumes to simulate the liquid film model (34) to (36). A first-order or a second-order Runge-Kutta time scheme may be used. The convective fluxes are approached with Rusanov numerical flux. The first-order space scheme uses the variables at the center of a cell whereas the second-order space scheme uses a classical MUSCL scheme (with minmod limiter) to rebuild $(h, \bar{u}, \bar{v})$ variables on both sides of cell interfaces. The pre-processing is carried out with the open-source solver Code_Saturne [34] and the post-processing with Gnuplot.

Verifications and validations are carried out on the model equations (37) and (38). This set of equations comes from the general model (34) to (36) with constant gas pressure, without rotational effect, surface tension and droplet impact and written in $1 \mathrm{D} \vec{y}$ direction. As the code is structurally $2 \mathrm{D}$, the same results were obtained in the 1D $\vec{x}$ direction.

$$
\begin{gathered}
\frac{\partial h}{\partial t}+\frac{\partial h \bar{v}}{\partial y}=S_{m}^{\prime} \\
(h \bar{v})_{, t}+\left(\Gamma h \bar{v}^{2}+\frac{g \cos \theta h^{2}}{2}\right)_{, y}=g \sin \theta h+\frac{1}{\rho}\left(\tau_{y z-\left.r\right|_{\eta}}-\tau_{y z-\left.r\right|_{0}}\right)
\end{gathered}
$$

$S_{m}^{\prime}$ is defined in the paragraph Model validation.

\section{Scheme verification}

First verifications are carried out on the model (39), which is the model equations (37) and (38) without shear and mass terms, with an analytical solution (Dam break) and with numerical results found by Leveque [35] (Hump) .

$$
\begin{aligned}
& (h)_{, t}+(h \bar{v})_{, y}=0 \\
& (h \bar{v})_{, t}+\left(\Gamma h \bar{v}^{2}+\frac{g \cos \theta h^{2}}{2}\right)_{, y}=g \sin \theta h
\end{aligned}
$$

Dam break The dam break initial conditions are zero velocity everywhere $(\bar{v}(y)=0)$, two constant height (left and right) such that $h_{\text {left }}>h_{\text {right }}$ and $\theta=0^{\circ}$. For $\Gamma=1$, an analytical solution with dam break initial conditions is found. The only possible evolution for the equation (39) consists in the succession of a rarefaction wave and a shock wave. Figure 5 compares the analytical solution given by the code at first and at second order. The Rusanov flux fits well the analytical solution even if there is numerical diffusion. The latter diminishes at second order. The results are mesh converged as shown in table 1. For the finer meshes, the order of convergence is close to 1 as expected for both first and second order schemes. For $\Gamma=6 / 5$, no analytical solution is found [9]. Figure 6 compares the code results for $\Gamma=1$ and $\Gamma=6 / 5$. The results have the same pattern but the $\Gamma=6 / 5$ result is shifted below and on the right with respect to
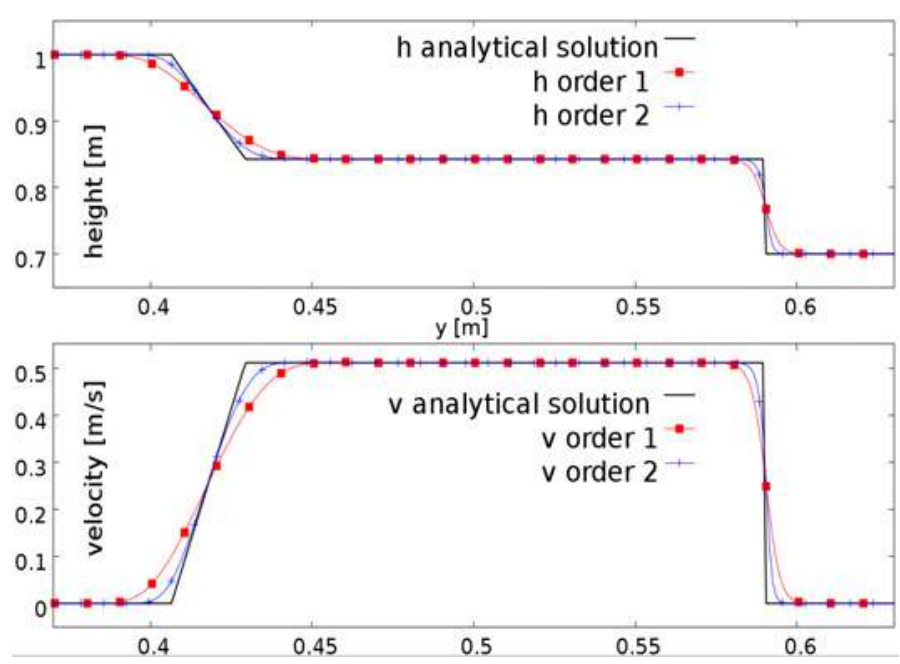

FIGURE 5. LIQUID FILM HEIGHT AND VELOCITY WITH INITIAL CONDITIONS $H_{L E F T}=1$ and $H_{R I G H T}=0.7$ AT T=0.03S AND WITH 1000 CELLS

TABLE 1. MESH CONVERGENCE FOR THE DAM BREAK WITH $\Gamma=1$

\begin{tabular}{|l|c|c|}
\hline Cells number N & $\mathscr{L}^{1}$ Error & $\alpha_{0}$ such that: error $(N)=a_{0}\left(\frac{1}{N}\right)^{\alpha_{0}}$ \\
\hline 100 & $2,3.10^{-2}$ & $/$ \\
\hline 1000 & $4,0.10^{-3}$ & 0,76 \\
\hline 10000 & $6,0.10^{-4}$ & 0,82 \\
\hline 100000 & $8,2.10^{-5}$ & 0,86 \\
\hline
\end{tabular}

the $\Gamma=1$ result. In this case, the results do not differ significantly for a linear and a parabolic velocity profile.

Hump As explained by Leveque [35], the evolution of the model (39) with $\Gamma=1, \theta=0^{\circ}$ and hump initial condition (Gaussian height and zero velocity) consists in two waves going in each direction. Each wave has a shock wave at its front and a rarefaction wave at its tail. Figure 7 shows numerical results displaying the expected features for a mesh including 10000 cells.

Inclined lake at rest The gravity term, $g \sin \theta h$ is now active $\left(\theta=10^{\circ}\right)$ in the model (39) with $\Gamma=1$. Analytically, the initial conditions representing an inclined lake at rest $(\bar{v}=0$ and $\left.h=\tan (\theta) y+h_{0}\right)$ satisfy the model (39). Indeed, the gravity source term compensates the convection variation. Numerically, the code predicts well this behaviour as the lake does not evolve. 


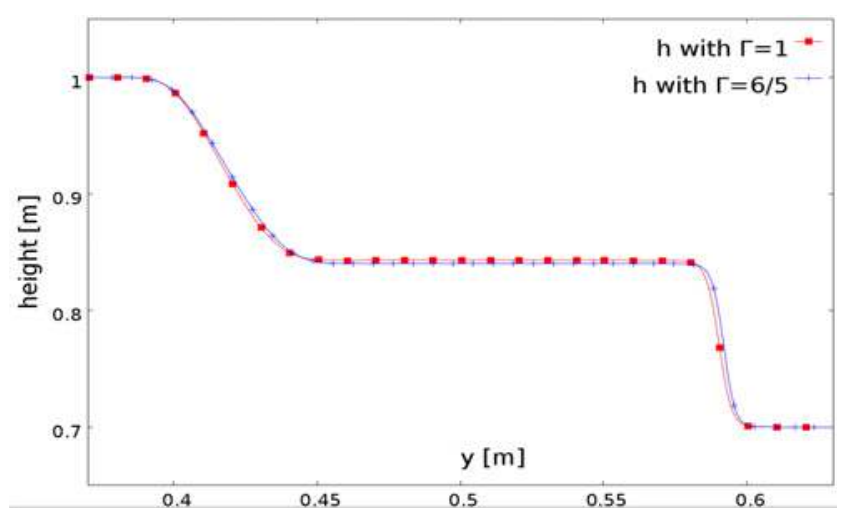

FIGURE 6. LIQUID FILM HEIGHT FOR DIFFERENT PROFILE FACTORS, $\Gamma$ AT T=0.03S AND WITH 1000 CELLS
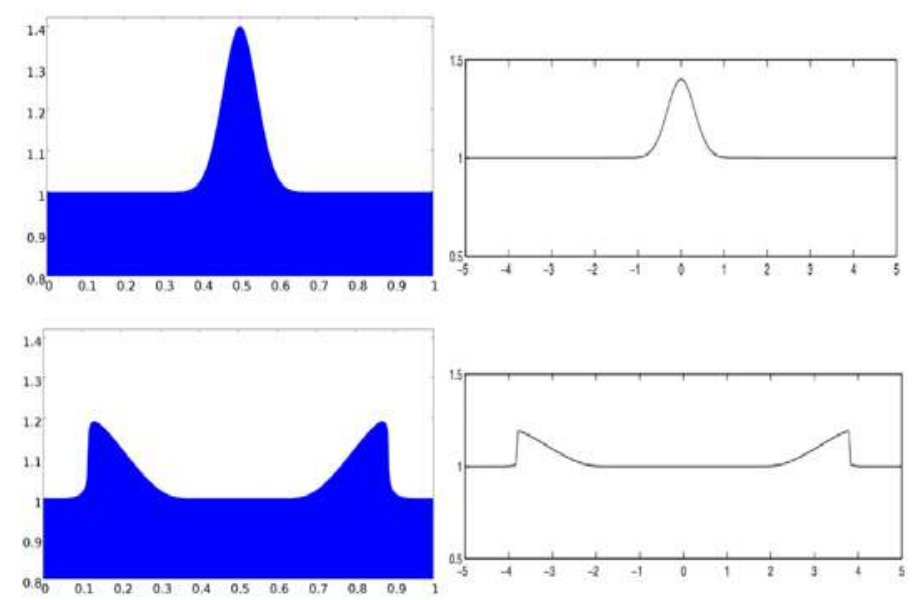

FIGURE 7. HEIGHT VS Y: HUMP CODE'S RESULTS (LEFT) AND LEVEQUE'S RESULT (RIGHT)

Falling liquid film A small perturbation on a falling liquid film on an inclined flat plate can either be amplified (become unstable) or be reduced (become stable). A theoretical linear stability analysis on the Navier-Stokes equations reveals that the critical Reynolds number (or the stability threshold) for long wave perturbations is $5 \operatorname{cotan}(\theta) / 6$. This value is validated with the experiment of Liu and Gollub [36]. To represent this situation, friction at the wall (expression (20)) is added to the model (39) which leads to the model (40):

$$
\begin{aligned}
& (h)_{, t}+(h \bar{v})_{, y}=0 \\
& (h \bar{v})_{, t}+\left(h \bar{v}^{2}+\frac{g \cos \theta h^{2}}{2}\right)_{, y}=g \sin \theta h-\frac{3 u v}{h}
\end{aligned}
$$

For the initial conditions, we have a constant velocity $(\bar{v}=$ 0.0546) and a long-wave sinusoidal height $\left(h=h_{0}(1+\right.$ $\left.\left.10^{-6} \sin (2 \pi y / 0.07)\right)\right)$. Table 2 gives numerical results with the
TABLE 2. NUMERICAL EXPERIMENT EQ. (40): UNSTABLE (U) AND STABLE (S) REGIME FOR DIFFERENT REYNOLDS

\begin{tabular}{|l|l|l|l|l|l|l|l|}
\hline Cells number/Re & 7.7 & 7.8 & 7.9 & 8.0 & 8.1 & 8.2 & 8.3 \\
\hline 100 & $\mathrm{~S}$ & $\mathrm{~S}$ & $\mathrm{~S}$ & $\mathrm{~S}$ & $\mathrm{~S}$ & $\mathrm{~S}$ & $\mathrm{U}$ \\
\hline 1000 & $\mathrm{~S}$ & $\mathrm{~S}$ & $\mathrm{U}$ & $\mathrm{U}$ & $\mathrm{U}$ & $\mathrm{U}$ & $\mathrm{U}$ \\
\hline 10000 & $\mathrm{~S}$ & $\mathrm{U}$ & $\mathrm{U}$ & $\mathrm{U}$ & $\mathrm{U}$ & $\mathrm{U}$ & $\mathrm{U}$ \\
\hline 100000 & $\mathrm{~S}$ & $\mathrm{U}$ & $\mathrm{U}$ & $\mathrm{U}$ & $\mathrm{U}$ & $\mathrm{U}$ & $\mathrm{U}$ \\
\hline
\end{tabular}

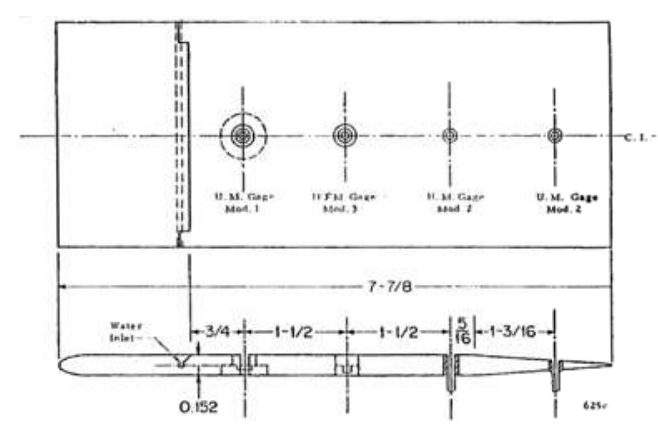

FIGURE 8. FLAT PLATE PROFILE USED IN THE EXPERIMENT OF HAMMITT'S ET AL. [1], [20], [3]

following experiment conditions [36]: angle of $6.4^{\circ}$ and kinematic viscosity of $6,28 \cdot 10^{-6} \mathrm{~m}^{2} / \mathrm{s}$. Further numerical experiment is needed to conclude since surface tension should be included and a linear stability analysis on the model (40) remains to be done. Nonetheless, for $\theta=6.4$, the numerical critical Reynolds number is less than 7.8 which is rather close to the experimental critical Reynolds number, 7.4.

\section{Model validation}

For a validation in low-pressure (LP) turbine conditions, the experimental work of Hammitt et al.'s [1] has been numerically reproduced. They conduct an experiment involving a sheared liquid film under LP steam turbine conditions. Water is pumped vertically through a gap on a horizontal flat plate. The created liquid film is sheared by high velocity steam near saturation. Both phases are approximately at 0.2 bar and $52.2{ }^{\circ} \mathrm{C}$. The height of the liquid film is measured at different positions on the flat plate as sketched in figure 8 .

To represent the experiment, we take into account gravity, friction at the wall and at the free surface (equations (37) and (38) with $\Gamma=1$ ). The properties of the liquid film and steam are calculated using IAPWS-IF97 formulations. We initialize the height and velocity at zero. At each time step, we introduce on a portion of the plate the equivalent flow $\left(5.10^{-7} \mathrm{~m}^{3} / \mathrm{s}\right)$ as a 


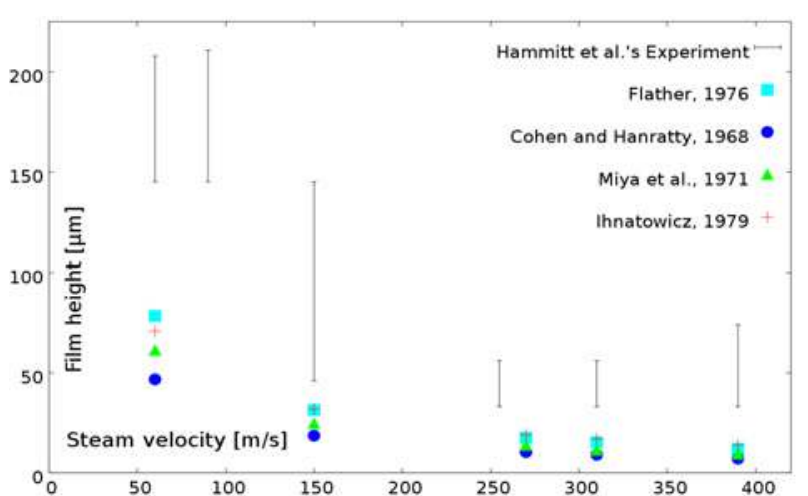

FIGURE 9. HAMMITT ET AL.'S EXPERIMENT [1] AND CODE RESULTS WITH WALL FRICTION EQ. (20)

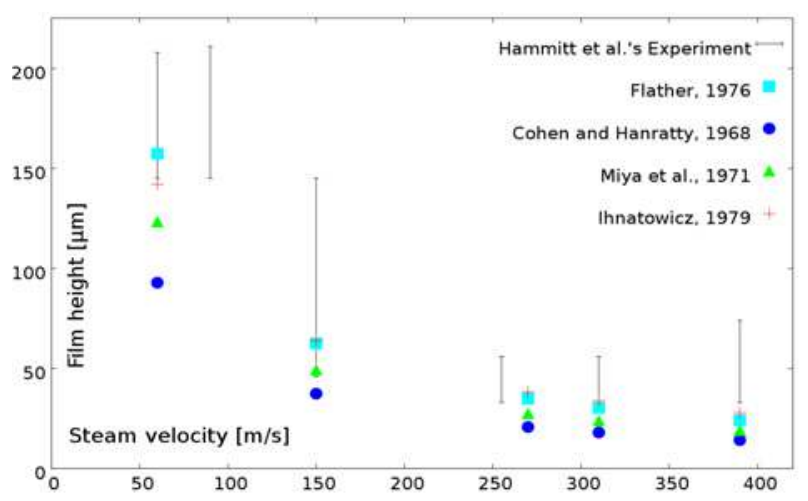

FIGURE 10. HAMMITT ET AL.'S EXPERIMENT [1] AND CODE RESULTS WITH WALL FRICTION EQ. (17) with EQ. (18)

mass source term, $S_{m}^{\prime}$ with no velocity. The results shown in figure 9,10 and 11 are mesh converged, they do not depend on the length of the portion on which we introduce the liquid, and are obtained for $C F L=0,06$. Indeed, the fact that the liquid film is less viscous for lower pressure and that the film is highly sheared, lead to a stability issue which is handled by lowering the CFL.

Figures 9, 10 and 11 give the experiment data (bar range) and compare the effect of the closure relations used for the wall friction ((17) with (18) and (19), and (20)) and for the free surface ((24) with (25), (26), (27), (28)). All the combinations of friction for the wall and the free surface give the same tendency: height decreases with steam velocity. On top of that, the combination of the friction wall equation (17) with (19) and the interfacial friction equation (24) with (28) predicts, for all measured steam velocities, the height of the film within the experiment uncertainty of measurements. The model is thus capable of describing accurately the height of a highly sheared

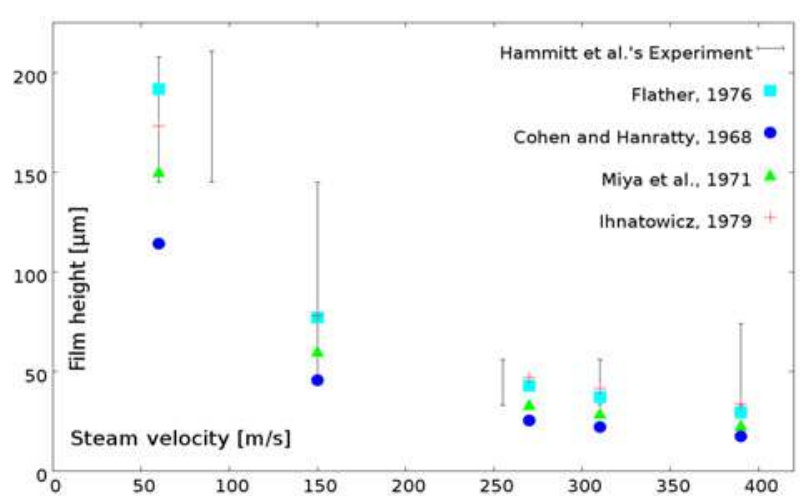

FIGURE 11. HAMMITT ET AL.'S EXPERIMENT [1] AND CODE RESULTS WITH WALL FRICTION EQ. (17) with EQ. (19)

liquid film on a flat plate using the expression of Spedding and Hand for the wall friction (17) with (19) and with the expression of Ihnatowicz (24) for the friction at the free surface.

\section{Summary and ongoing work}

In this paper, a model for liquid films on steam turbine stator and rotor blades based on an integral formulation and on a rich description of physical phenomena has been presented. The required closure laws are detailed. The implementation of the convective part has been verified with two Riemann problems. In the dam break one, no significant variations were observed between a vertical and a parabolic velocity profile. The gravity source term implementation has been verified with the analytical solution of an inclined lake at rest. The friction at the wall has been investigated with a falling liquid film case. A linear stability analysis is still needed to assess stability criteria. Several frictions laws at the wall and at the free surface have been compared with the experiment on a sheared liquid film under low-pressure steam turbine conditions. In this case, the use of Spedding and Hand [15] wall friction model and Ihnatowicz [23] free surface friction model gives the better numerical results in terms of agreement with the experiment.

The model and the 2D code, which has been developed, show encouraging results but further steps are to be achieved to reach a full simulation of the liquid film in steam turbine. As liquid films in steam turbine can be under inverted gravity, this situation will be tested and compared with the experiment of Brun et al. [37]. Surface tension and capillarity effects will be compared with the experiment of Liu and Gollub [36]. Then, the gas pressure, the droplet impact and the rotational effect will be added to the code. Moreover, degrees of freedom will be given to the position of the flat plate and the inclusion of the blade curvature will be investigated to be representative of turbine blades. Finally, wet steam fields obtained for an industrial steam turbine case [10], [14] will be used as an input to the code 2D to simulate a representative film on steam turbines blades. 


\section{REFERENCES}

[1] Hammitt, F. G., Hwang, J.-B. G., Mancuso, A., Krause, D., and Blome, S., 1975. "Liquid film thickness tests: wet steam tunnel". Technical Report UMICH 012449-9-T, Michigan University.

[2] Stanciu, M., Marcelet, M., and Dorey, J.-M. "Numerical investigation of condenser pressure effect on last stage operation of low pressure wet steam turbines". In Turbo Expo 2013, American Society of Mechanical Engineers.

[3] Hammitt, F. G., et al., 1981. "Liquid film and droplet stability consideration as applied to wet steam flow". Forschung im Ingenieurwesen A, 47(1), pp. 1-14.

[4] Gyarmathy, G., 1962. "Bases of a theory for wet steam turbines". PhD thesis, Diss. Techn. Wiss. ETH Zürich.

[5] Kirillov, and Yablonik, 1970. Fundamentals of the theory of turbines operating on wet steam. National Aeronautics and Space Administration TT F-611.

[6] Malamatenios, C., Giannakoglou, K., and Papailiou, K., 1994. "A coupled two-phase shear layer/liquid film calculation method. formulation of the physical problem and solution algorithm". International journal of multiphase flow, 20(3), pp. 593-612.

[7] Williams, J., and Young, J. B., 2007. "Movement of deposited water on turbomachinery rotor blade surfaces". Journal of turbomachinery, 129(2), pp. 394-403.

[8] Schuster, S., Benra, F.-K., Dohmen, H. J., König, S., and Martens, U. "Sensitivity analysis of condensation model constants on calculated liquid film motion in radial turbines". In Turbo Expo 2014, American Society of Mechanical Engineers.

[9] Simon, A. 2017, Ecole Centrale de Lyon, in preparation.

[10] Fendler, Y., 2012. "Analyse des phénomènes liés à la présence de la phase liquide dans les turbines à vapeur et élaboration de modèles méridiens pour en prédire les effets". PhD thesis, Ecole Centrale de Lyon, https://tel.archives-ouvertes.fr/tel-00818336.

[11] Wehausen, J. V., and Laitone, E. V., 1960. Surface waves. Springer.

[12] Miya, M., Woodmansee, D. E., and Hanratty, T. J., 1971. "A model for roll waves in gas-liquid flow". Chemical Engineering Science, 26(11), pp. 1915-1931.

[13] Ruyer-Quil, C., and Manneville, P., 1998. "Modeling film flows down inclined planes". The European Physical Journal BCondensed Matter and Complex Systems, 6(2), pp. 277-292.

[14] Blondel, F., 2014. "Couplages instationnaires de la vapeur humide dans les écoulements de turbines à vapeur". $\mathrm{PhD}$ thesis, Ecole Centrale de Lyon, https://tel.archives-ouvertes.fr/tel-00985725.

[15] Spedding, P., and Hand, N., 1997. "Prediction in stratified gasliquid co-current flow in horizontal pipelines". International journal of heat and mass transfer, 40(8), pp. 1923-1935.

[16] Stanton, D. W., and Rutland, C. J., 1998. "Multi-dimensional modeling of thin liquid films and spray-wall interactions resulting from impinging sprays". International Journal of Heat and Mass Transfer, 41(20), pp. 3037-3054.

[17] Foucart, H., 1999. "Modélisation tridimensionnelle des films liquides pariétaux dans les moteurs à combustion interne". $\mathrm{PhD}$ thesis, Université de Rouen.

[18] Lilleleht, L. U., and Hanratty, T. J., 1961. "Measurement of interfacial structure for co-current air-water flow". Journal of Fluid Mechanics, 11(01), pp. 65-81.
[19] Chang, F., and Dukler, A., 1964. "The influence of a wavy, moving interface on pressure drop for flow in conduits". International Journal of Heat and Mass Transfer, 7(12), pp. 1395-1404.

[20] Kim, W., 1978. "Study of liquid films, fingers, and droplet motion for steam turbine blading erosion problem". Technical Report UMICH 014571-4-T, Michigan University.

[21] Flather, R. A., 1976. "Results from a storm surge prediction model of the north-west european continental shelf for april, november and december, 1973". Technical Report 24, Wormley, UK, Institute of Oceanographic Sciences.

[22] Cohen, L. S., and Hanratty, T. J., 1968. "Effect of waves at a gas-liquid interface on a turbulent air flow". Journal of Fluid Mechanics, 31(03), pp. 467-479.

[23] Ihnatowicz, E., Gumkowski, S., and Mikielewicz, J., 1979. "Experimental study of evaporation and breakdown of thin liquid films driven by shear stresses". Journal of Heat Transfer, 101(4), pp. 712-717.

[24] MJ, and Sculpher, P., 1969. "Paper 19: Conditions producing concentrated erosion in large steam turbines". In Proceedings of the Institution of Mechanical Engineers, Conference Proceedings, Vol. 184, SAGE Publications, pp. 45-56.

[25] Crane, R., 1973. "Deposition of fog drops on low pressure steam turbine blades". International Journal of Mechanical Sciences, 15(8), pp. 613-631.

[26] Young, J., and Yau, K., 1988. "The inertial deposition of fog droplets on steam turbine blades". Journal of turbomachinery, 110(2), pp. 155-162.

[27] Williams, G., and Lord, M., 1976. "Measurement of coarse water distribution in the lp cylinders of operating steam turbines". Proceedings of the Institution of Mechanical Engineers, 190(1), pp. 59-69.

[28] Zaichik, L., Nigmatulin, B., and Pershukov, V., 1995. "Modelling of dynamics of aerosols in near-wall turbulent flows and particle deposition in pipes". In Proceedings of the 2nd International Conference on Multiphase Flow, Vol. 4, pp. 9-16.

[29] Godlewski, E., and Sainte-Marie, J., 2015. (Not so) hyperbolic models for complex flows Application to sustainable energie.

[30] Bertschy, J., Chin, R., and Abernathy, F., 1983. "High-strain-rate free-surface boundary-layer flows". Journal of Fluid Mechanics, 126, pp. 443-461.

[31] Alekseenko, S., Nakoryakov, V. Y., and Pokusaev, B., 1985. "Wave formation on a vertical falling liquid film". AIChE Journal, 31(9).

[32] Adomeit, P., and Renz, U., 2000. "Hydrodynamics of threedimensional waves in laminar falling films". International journal of multiphase flow, 26(7), pp. 1183-1208.

[33] Dellar, P. J., and Salmon, R., 2005. "Shallow water equations with a complete coriolis force and topography". Physics of Fluids, 17(10), p. 106601.

[34] Code_saturne web site: http://code-saturne.org/cms/.

[35] LeVeque, R. J., 2002. Finite volume methods for hyperbolic problems, Vol. 31. Cambridge university press.

[36] Liu, J., and Gollub, J. P., 1994. "Solitary wave dynamics of film flows". Physics of Fluids, 6(5), pp. 1702-1712.

[37] Brun, P.-T., Damiano, A., Rieu, P., Balestra, G., and Gallaire, F., 2015. "Rayleigh-taylor instability under an inclined plane". arXiv preprint arXiv:1503.02345. 